\title{
磺化聚醚砜/薄水铝石复合质子交换膜的性能
}

\author{
文 胜 ${ }^{1,3}$ 龚春丽 $^{3}$ 蔡芳昌 ${ }^{1}$ 叶正涛 ${ }^{1,2, *}$ \\ ('湖北大学材料科学与工程学院, 武汉 430062; 2武汉纺织大学化学与化工学院, 武汉 430073; \\ 3 孝感学院化学与材料科学学院, 湖北孝感 432000)
}

\begin{abstract}
摘要: 为了提高膜的阻醇性能和高温下的质子传导性, 在磺化聚梄砜(SPES)中掺杂一种吸湿性的无机物 $\mathrm{AIOOH}$, 制备了一种新型的 SPES/AIOOH复合质子交换膜. 并经傅里叶变换红外(FTIR)光谱、热失重(TGA)、扫 描电镜(SEM)等手段对膜的结构和性能进行了表征. 结果表明: 复合膜较纯 SPES 膜具有更高的热稳定性和吸 水率; SEM 图片显示 $\mathrm{AlOOH}$ 在膜中分布均匀. 复合膜在高温下具有良好的质子传导性, 掺杂量为 $10 \%(w)$ 的复 合膜在 $120^{\circ} \mathrm{C}$ 下的质子传导率仍可保持在 $0.014 \mathrm{~S} \cdot \mathrm{cm}^{-1}$ 左右; 随着 $\mathrm{AlOOH}$ 含量的增加, 复合膜的阻醇性能大 大提高, 这表明该复合膜在直接甲醇燃料电池中具有良好的应用前景.
\end{abstract}

关键词：磺化聚醚砜; $\mathrm{AlOOH}$; 复合膜; 直接甲醇燃料电池 中图分类号: 0646

\section{Properties of SPES/AIOOH Composite Proton Exchange Membranes}

\author{
WEN Sheng ${ }^{1,3} \quad$ GONG Chun-Li $i^{3} \quad$ TSAI Fang-Chang ${ }^{1} \quad$ YEH Jen-Taut ${ }^{1,2,{ }^{*}}$ \\ ('Faculty of Material Science and Engineering, Hubei University, Wuhan 430062, P. R. China; ${ }^{2}$ Faculty of Chemistry and \\ Chemical Engineering, Wuhan Textile University, Wuhan 430073, P. R. China; ${ }^{3}$ Faculty of Chemistry and Materials Science, \\ Xiaogan University, Xiaogan 432000, Hubei Province, P. R. China)
}

\begin{abstract}
Novel sulfonated polyether sulfone (SPES)/AIOOH organic/inorganic composite membranes were prepared by doping SPES with $\mathrm{AlOOH}$, which lowered the methanol crossover and increased the proton conductivity at high temperatures. The structure and performance of the obtained membranes were characterized by Fourier transform infrared (FTIR) spectroscopy, thermogravimetric analysis (TGA), and scanning electron microscopy (SEM) etc. Compared with the pure SPES membrane the composite membranes had higher thermal stability and water uptake. The morphology of the composite membranes indicated that $\mathrm{AIOOH}$ was uniformly distributed throughout the SPES matrix. The network-like structure began to form when the $\mathrm{AlOOH}$ content was around $10 \%$. The proton conductivity was still ca $0.014 \mathrm{~S} \cdot \mathrm{cm}^{-1}$ even at a temperature as high as $120^{\circ} \mathrm{C}$. Additionally, the methanol resistance improved greatly as the content of $\mathrm{AlOOH}$ increased. The SPES/AIOOH composite membrane is a promising candidate for direct methanol fuel cell (DMFC) applications.
\end{abstract}

Key Words: Sulfonated poly(ether sulfone); $\mathrm{AlOOH}$; Composite membrane; Direct methanol fuel cell

\section{1 引言}

直接甲醇燃料电池(DMFC)结构简单、体积小、 比能量高、能量转化率高, 燃料甲醇来源丰富、储存
和携带方便、价格低廉, 是民用和军用便携电源及 电动交通工具的理想动力源. ${ }^{1,2}$ 膜电极是 $\mathrm{DMFC}$ 的 核心, 质子交换膜是膜电极的关键组成部分, ${ }^{3,4}$ 理想

Received: March 1, 2011; Revised: April 15, 2011; Published on Web: June 8, 2011.

${ }^{*}$ Corresponding author. Email: jyeh@mail.ntust.edu.tw; Tel: +86-712-2345464.

The project was supported by the Natural Science Foundation of Hubei Province, China (2010CDB00301).

湖北省自然科学基金(2010CDB00301)资助项目

(C) Editorial office of Acta Physico-Chimica Sinica 
的质子交换膜具有导通质子、阻止甲醇和氧化剂透 过的作用. 在 PEMFC 中广泛使用的全氟磺酸型膜, 如 Dupont 公司开发的 Nafion 系列膜, 在用于 DMFC 时同样具有优异的性能, 但遗憾的是其在 DMFC 中 应用时无法有效地阻挡甲醇向阴极渗透而致使电 池性能大大下降, 这是 DMFC 研究的两大主要挑战 之一(另一个大的难题是目前所使用的催化剂对甲 醇的催化氧化活性很低), ${ }^{1}$ 为了克服全氟磺酸型膜 在 DMFC 中应用的缺点, 提高电池性能, 国内外许 多研究者一直在致力于开发各种低透醇的质子交 换膜, 开发新材料的 DMFC 用质子交换膜是当今研 究的热点. 与此同时, 还在研发可在高温下(如高于 $100{ }^{\circ} \mathrm{C}$ ) 能稳定工作的质子交换膜, 当 DMFC 在较高 温度下工作时, 不仅可以提高甲醇在阳极的电化学 氧化速度, 还能降低阳极与膜界面处的醇浓度, 从 而减小了甲醇由膜的阳极侧向膜的阴极侧的迁移 量, 提高 DMFC 性能. ${ }^{6}$

聚醚砜是一种耐热性高、化学稳定性好、机械 性质稳定以及成膜性能优异的特种工程塑料, 采用 磺化剂对其进行磺化所得到具有质子传导能力的 磺化聚醚砜(SPES)引起了广泛的关注..$^{-10}$ 但其阻醇 性能和在高温下的质子传导性还不尽如人意, 通过 有机-无机复合是改善膜综合性能的有效途径之一. 许多研究表明, 掺杂吸湿性的无机氧化物不仅可以 大大提高膜的保水性能, 而且能够有效地阻止甲醇 的透过. Tsai 等 ${ }^{11}$ 将 $\mathrm{SiO}_{2}$ 掺入磺化聚芳醚砜共聚物 中, 制得了一种纳米复合膜, 通过测定单电池性能 发现该复合膜在 $80^{\circ} \mathrm{C}$ 时的单电池性能与 $\mathrm{Nafion}^{\circledR}$ 117 膜相近. $\mathrm{Su}$ 等 ${ }^{12}$ 以磺化二氧化硅 $\left(\mathrm{SiO}_{2}-\mathrm{SO}_{3} \mathrm{H}\right)$ 作 为掺杂体加入磺化聚杂荎联苯醚酮(SPPEK)中, 以 弥补纯 $\mathrm{SiO}_{2}$ 掺杂所造成的离子交换容量(IEC)值 的损失. 体系中 $\mathrm{SiO}_{2}-\mathrm{SO}_{3} \mathrm{H}$ 与 $\mathrm{SPPEK}$ 之间 $-\mathrm{SO}_{3} \mathrm{H} /$ $-\mathrm{SO}_{3} \mathrm{H}$ 的强烈相互作用形成了一种离子交联, 致使 复合膜的热稳定性和阻醇性能大大提高, 并且质子 传导率也明显增加, $\mathrm{SiO}_{2}-\mathrm{SO}_{3} \mathrm{H}$ 含量为 $7.5 \%(w)$ 的复 合膜的质子传导率是纯 SPPEK膜的 3.6 倍. Nunes 等 ${ }^{13}$ 分别以钛酸四乙酯和异丙醇锆为前驱体, 制备 了相界面形貌良好的磺化聚醚酮 $(\mathrm{SPEK}) / \mathrm{TiO}_{2}$ 、 $\mathrm{SPEK} / \mathrm{ZrO}_{2}$ 杂化膜材料, 阻醇性得到了大幅度提高, 但是质子传导率有所降低. 为实现质子传导率与阻 醇性的平衡, 在杂化膜中掺杂了无机质子导体材料 $\mathrm{Zr}\left(\mathrm{HPO}_{4}\right)_{2}$, 制备了 $\mathrm{SPEK} / \mathrm{Zr}\left(\mathrm{HPO}_{4}\right)_{2} / \mathrm{ZrO}_{2}$ 复合膜, 甲 醇渗透率可降至 $70 \mathrm{~g} \cdot\left(\mathrm{h} \cdot \mathrm{m}^{2}\right)^{-1}$, 而质子传导率与纯
SPEK 相比仅有 $10 \%$ 的损失. ${ }^{14}$ Shen 等 ${ }^{15}$ 在 PVDF-gPAMPS 中加入纳米 $\mathrm{Al}_{2} \mathrm{O}_{3}$, 同时提高了膜的质子传 导率和阻醇性能. $\mathrm{AlOOH}$ 是氧化铝的一水化合物, 也称为勃姆石或薄水铝石, 被广泛用作裂化、加氢 或重整催化剂的粘结剂或催化剂载体. ${ }^{16,17} \mathrm{AlOOH}$ 是 由氧原子密堆积的八面体联结在一起形成的复杂 层状结构, 结构中含有大量的羟基, 具有优异的吸 湿性能, 本文将 $\mathrm{AlOOH}$ 与 SPES 进行复合制备了 SPES/AlOOH复合膜, 并对膜的结构和性能进行了 研究, 探讨了其作为质子交换膜用于 DMFC 的可 能性.

\section{2 实验部分}

\section{1 试剂和仪器}

聚醚砜(PES): 德国 BASF 公司, Ultrason E6020P, $M_{\mathrm{w}}=58000 ; \mathrm{AlOOH}$ : 山东铝业公司, P-DF-09-HSi, 比 表面积 $S_{\mathrm{BET}}=320 \mathrm{~m}^{2} \cdot \mathrm{g}^{-1}$, 体积空隙率为 $0.9-1.2 \mathrm{~mL}$. $\mathrm{g}^{-1} ; \mathrm{N}, \mathrm{N}$-二甲基乙酰胺(DMAc): 分析纯, 国药集团 化学试剂有限公司; 浓硫酸: 分析纯, 开封东大化工 有限公司; 氯磺酸: 化学纯, 中国亭新化工试剂厂.

FTIR 光谱: 美国 Thermoelectron 公司 Nicolet 380; TGA: 美国 TA 公司 SDT-Q600; 示差扫描量热 (DSC): 美国 TA 公司 DSC Q-100; SEM: 日本 HITACHI X-650; X 射线能谱分析仪: 英国 Oxford仪器公 司 X能谱(EDX) 1350; X射线衍射分析(XRD): 日本 理学公司 D/max-IIIc; 气相色谱仪: 美国 HP 公司 GC-5890 seriesII; 频率响应分析仪: 英国 Solatron 公 司 1260 .

\section{2 聚醚砜的磺化与复合膜的制备}

聚醚砜的磺化以浓硫酸为溶剂, 氯磺酸为磺化 剂来进行, 具体步骤参照文献. ${ }^{8}$ IEC 值的测定采用 酸碱滴定法, 将 SPES 膜样品浸泡在大量的 $1 \mathrm{~mol}$. $\mathrm{L}^{-1}$ 的 $\mathrm{NaCl}$ 溶液中至少 $48 \mathrm{~h}$, 使样品中的磺酸基团 都交换成钠型, 然后用 $0.01 \mathrm{~mol} \cdot \mathrm{L}^{-1}$ 的 $\mathrm{NaOH}$ 标准溶 液来滴定浸泡后液体中氢离子. IEC 值由下面公式 来进行计算:

$$
\mathrm{IEC}=\frac{c_{\mathrm{NaOH}} \times V_{\mathrm{NaOH}}}{m_{\mathrm{SPES}}}
$$

其中 $c_{\mathrm{NaOH}}$ 是 $\mathrm{NaOH}$ 溶液的浓度 $\left(\mathrm{mol} \cdot \mathrm{L}^{-1}\right), V_{\mathrm{NaOH}}$ 是所 用 $\mathrm{NaOH}$ 溶液的体积 $(\mathrm{mL}), m$ 是 SPES 的质量 $(\mathrm{g})$. 实 验得到 SPES 的磺化度为 $37 \%$, 对应的 IEC 值为 $1.41 \mathrm{mmol} \cdot \mathrm{g}^{-1}$.

室温下将 SPES 溶于 DMAc 形成 $6 \%(w)$ 的均相 
溶液待用. 称取一定量的 $\mathrm{AlOOH}$ 加入已配好的 SPES 溶液中, 磁力摚拌 $2 \mathrm{~h}$, 将混合物倒入自制的成 膜板中, 置于烘箱, $60^{\circ} \mathrm{C}$ 烘 $4 \mathrm{~h}, 80^{\circ} \mathrm{C}$ 烘 $10 \mathrm{~h}, 100^{\circ} \mathrm{C}$ 烘 $4 \mathrm{~h}$. 最后待自然冷却后揭膜, 复合膜用 SPES/ $\mathrm{AlOOH} x$ 表示, 其中 $x$ 为 $\mathrm{AlOOH}$ 占 SPES 的摩尔比.

\section{3 复合膜结构与性能表征}

$\mathrm{AlOOH}$ 的FTIR 测试采用 $\mathrm{KBr}$ 压片法, SPES 和 复合膜的 FTIR 谱利用 ATR 附件直接对膜样品进行 测试, 分辨率为 $4 \mathrm{~cm}^{-1}$.

膜的热稳定性采用热重分析仪进行测试, 温度 为室温 $\left(25^{\circ} \mathrm{C}\right)-700{ }^{\circ} \mathrm{C}$, 升温速率 $10^{\circ} \mathrm{C} \cdot \mathrm{min}^{-1}, \mathrm{~N}_{2}$ 保 护.

$\mathrm{XRD}$ 分析扫描范围为 $5^{\circ}-80^{\circ}$, 扫描速率 $3\left(^{\circ}\right)$. $\mathrm{min}^{-1}$. 膜的吸水率就是干膜吸水的质量比率, 将一 定质量的干膜浸泡于去离子水中, $24 \mathrm{~h}$ 后取出, 用滤 纸迅速吸去膜表面的水分后准确称取其质量, 其含 水率可根据公式(2)计算:

$$
\text { 含水率 }\left(w_{\mathrm{t}}\right)=\frac{m_{\mathrm{w}}-m_{\mathrm{d}}}{m_{\mathrm{d}}} \times 100 \%
$$

式中, $m_{\mathrm{w}}$ 为膜在室温下的湿重 $(\mathrm{g}) ; m_{\mathrm{d}}$ 为膜在室温下 的干重 $(\mathrm{g})$.

溶胀性测试: 将膜在 $100{ }^{\circ} \mathrm{C}$ 干燥至恒重, 冷却 后量取膜的长、宽、厚尺寸, 然后将膜放入 $100{ }^{\circ} \mathrm{C}$ 去 离子水中浸泡 $24 \mathrm{~h}$, 取出膜, 快速量取湿膜的尺寸, 由下式计算出膜的尺寸变化率:

$$
\Delta l=\frac{l_{\mathrm{w}}-l_{\mathrm{d}}}{l_{\mathrm{d}}} \times 100 \%
$$

式中, $l_{\mathrm{w}}$ 为膜处于湿态时的长度; $l_{\mathrm{d}}$ 为膜处于干态下 的长度. 宽度和厚度的尺寸改变也如式(3)进行求 取.

采用 SEM 观察了膜的表面和断裂面微结构. 其 中, 加速电压为 $15 \mathrm{kV}$, 样品在液氮下淬断, 断面喷 金后观察, 同时将膜的断面在 X射线能谱分析仪上 进行 EDX 分析.

分别对不同比例的复合膜进行了氧化稳定性 测试, 将膜放在 $80^{\circ} \mathrm{C}$ 含有 $2 \times 10^{-6}(w) \mathrm{FeSO}_{4}$ 的 $3 \%$ (w) $\mathrm{H}_{2} \mathrm{O}_{2}$ 溶液中 (此种溶液称为 Fenton 溶液), 通过记 录膜开始溶解的时间作为评价其氧化稳定性的依 据.

将膜固定在自制的电导率测试平台上, 将此平 台置于恒温恒湿箱中, 测试台上开有一个方孔, 这 样可使置于其中的膜与环境的温度、湿度保持一 致. 膜的电导率采用两电极交流阻抗法在频率响应
分析仪上测定, 频率扫描范围为 $1-10^{7} \mathrm{~Hz}$, 交流信号 振幅为 $100 \mathrm{mV}$. 在测试之前, 膜样品置于室温去离 子水中达饱和. 膜的质子传导率 $\sigma\left(\mathrm{S} \cdot \mathrm{cm}^{-1}\right)$ 通过下式 进行计算:

$$
\sigma=\frac{a}{R \times A}
$$

式中, $a$ 和 $A$ 分别为两电极的间距和两电极间待测 膜的有效横截面积, $R$ 是膜的平面方向的阻抗, 通过 Nyquist 图中曲线与 $x$ 轴在高频端的截距求得.

采用隔膜扩散池来测试膜的阻醇性能. 将膜夹 在 $\mathrm{A} 、 \mathrm{~B}$ 两半室中间, 在 $\mathrm{A}$ 侧注入 $20 \mathrm{~mL}$ 含有甲醇和 乙醇体积分数为 $8 \%$ 和 $0.2 \%$ 的水溶液, $\mathrm{B}$ 侧注入同 体积的乙醇体积分数为 $0.2 \%$ 的水溶液. 利用气相色 谱仪来检测另一侧甲醇浓度的变化, 其中色谱柱采 用的是 HP-20M (CARBOWAX 20M phase)毛细管色 谱柱, 隔膜池中两侧均含有乙醇是为了作内标. 膜 的甲醇渗透系数采用下式进行计算:

$$
P_{\mathrm{m}}=\frac{c_{\mathrm{B}} \times V_{\mathrm{B}} \times L}{c_{\mathrm{A}} \times A \times t}
$$

式中, $c_{\mathrm{B}}$ 是 $t$ 时刻 $\mathrm{B}$ 侧的甲醇浓度, $c_{\mathrm{A}}$ 是 $\mathrm{A}$ 侧的甲醇 浓度, $A 、 L$ 和 $V_{\mathrm{B}}$ 分别是膜的面积、厚度和 $\mathrm{B}$ 侧溶液的 体积, $t$ 为渗透的时间, $P_{\mathrm{m}}$ 为渗透系数.

\section{3 结果与讨论}

\subsection{FTIR 光谱}

图 1 为 $\mathrm{AlOOH} 、 \mathrm{SPES}$ 及复合膜的红外光谱图, $\mathrm{AlOOH}$ 中 $1074 \mathrm{~cm}^{-1}$ 处的强峰和 $1160 \mathrm{~cm}^{-1}$ 处的肩峰 分别归属于 $\mathrm{Al}-\mathrm{O}-\mathrm{H}$ 的对称弯曲振动和非对称弯 曲振动, 743、616和 $483 \mathrm{~cm}^{-1}$ 处的吸收带分别对应于

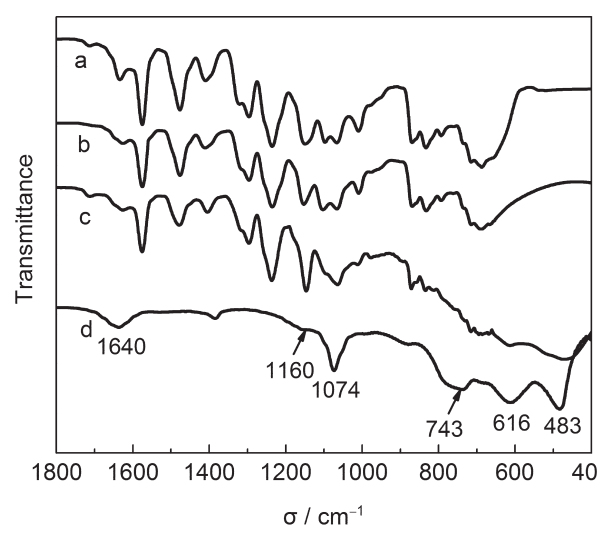

图 $1 \mathrm{AlOOH}$ 和 SPES/AIOOH 复合膜的 FTIR 光谱图

Fig.1 FTIR spectra of AIOOH and SPES/AIOOH composite membranes

(a) SPES, (b) SPES/AlOOH 5\% (w), (c) SPES/AIOOH 10\% (w), (d) AlOOH; SPES: sulfonated polyether sulfone 
$\mathrm{Al}-\mathrm{O}$ 的扭曲振动、伸缩振动和弯曲振动. ${ }^{18}$ 对于纯 SPES, $1066 \mathrm{~cm}^{-1}$ 处的吸收峰是属于磺酸基团的对称 伸缩振动, $1007 \mathrm{~cm}^{-1}$ 对应于磺酸基团中 $\mathrm{S}-\mathrm{O}$ 键的 伸缩振动峰, 1362、1237 和 $1275 \mathrm{~cm}^{-1}$ 归属于芳醚 $\mathrm{C}-\mathrm{O}-\mathrm{C}$ 的伸缩振动, 而 1446-1630 $\mathrm{cm}^{-1}$ 波段的吸 收峰则属于芳环骨架的伸缩振动. 当 SPES 中掺入 $5 \%(w)$ 的 $\mathrm{AlOOH}$ 后, 其 FTIR 图谱与纯 SPES 相比并 无太大变化, 但对于 SPES/AlOOH 10\% $(w)$ 复合膜而 言, 1066 和 $1100 \mathrm{~cm}^{-1}$ 处的吸收峰由双峰变成了一个 宽峰, 而 400-800 $\mathrm{cm}^{-1}$ 范围内的吸收带的形状和强 度也发生了较大改变, 出现了 $\mathrm{Al}-\mathrm{O}$ 键的特征吸收 峰, 这表明掺杂的 $\mathrm{AlOOH}$ 与 SPES 得到了较好的复 合.

\section{2 热失重分析}

图 2 分别为 $\mathrm{AlOOH}$ 和 SPES/AlOOH复合膜的 TG-DTG 曲线, $\mathrm{AlOOH}$ 在整个受热区间的失重过程 有两个阶段: 第一阶段是室温 $\left(25^{\circ} \mathrm{C}\right)-200^{\circ} \mathrm{C}$, 对应 于样品表面吸附水和内部容留的水的逐步脱除; 在 $400-550{ }^{\circ} \mathrm{C}$ 区间的失重是 $\mathrm{AlOOH}$ 中羟基的完全脱 除, 形成氧化铝: $2 \mathrm{AlOOH}=\mathrm{Al}_{2} \mathrm{O}_{3}+\mathrm{H}_{2} \mathrm{O}$, 这与文献 ${ }^{19}$ 所 报道的基本相符. 对于 SPES/AlOOH 10\%复合膜而 言, 其与纯 SPES 的 TG 曲线较为类似, 出现了三个 阶段的失重区间, 第一阶段位于 $150-250{ }^{\circ} \mathrm{C}$, 失重 量很小 (约 $2.5 \%)$, 这可能与膜中由于氢键所吸附的 水和残留溶剂 DMAc 有关. ${ }^{2,20}$ 第二阶段的降解温度 开始于 $380^{\circ} \mathrm{C}$, 这应该归属于磺酸基团的降解和所 掺杂的 $\mathrm{AlOOH}$ 中羊基的脱除. 第三阶段是主链的降 解所引起的失重. 从 DTG 曲线还可以明显看出, $\mathrm{AlOOH}$ 的加入导致聚合物主链的降解温度明显向 高温移动, 这表明复合膜的热稳定性得到了一定程

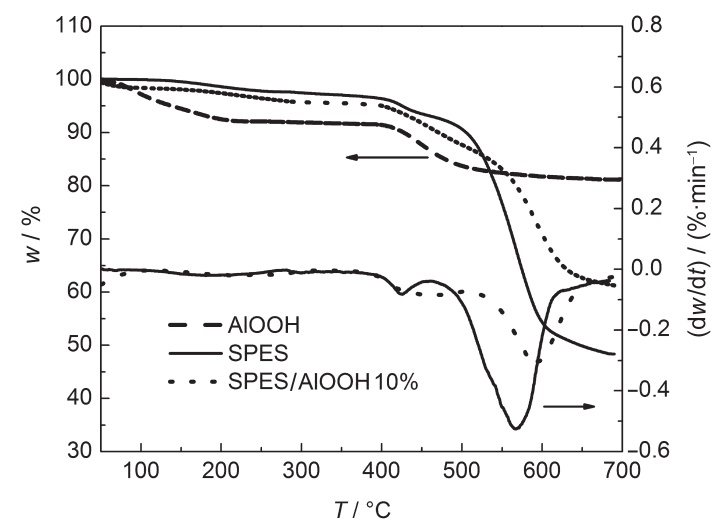

图 $2 \mathrm{AlOOH}$ 和复合膜的热重-微商热重(TG-DTG)曲线

Fig.2 Thermalgravimetric-derivated thermalgravimetric (TG-DTG) curves of AlOOH and composite membranes
度的提高.

\subsection{X 射线衍射分析}

AlOOH、SPES 膜和 SPES/AlOOH 复合膜的 X 射线衍射图如图 3 所示, 由图 3 中(c)可见, $\mathrm{AlOOH}$ 中 $2 \theta=14.4^{\circ}, 28.0^{\circ}, 38.3^{\circ}, 48.9^{\circ}, 49.2^{\circ}, 64.9^{\circ}$ 的几个主要 衍射峰分别对应(020)、(120)、(031)、(200)、(051)和 (002)晶面的衍射, 各个衍射峰及相应的晶面指数与 勃姆石 AlOOH 的 PDF 卡片完全吻合 (JCPDS 211307). 对于 SPES 而言, 只有在 $2 \theta=18^{\circ}$ 附近有一个大 而宽的弥散峰, 这表明 SPES 完全处于无定形态, 当 掺入 $\mathrm{AlOOH}$ 后, 在复合膜的 XRD 图中出现了(120)、 (031)、(200)、(051)这些 AlOOH 所特有晶面的衍射 峰, 另外 $2 \theta=18^{\circ}$ 处的弥散峰变窄且强度有所减弱, 这些结果表明 AlOOH 的引入使得 SPES 的有序性有 所降低.

\section{4 复合膜的形态结构分析}

利用 SEM 对复合膜的表面和断面形态结构进 行了观察. 由图 4(a)和 $\left(a^{\prime}\right)$ 可以看出, 纯 SPES 膜的表 面致密、光滑和均匀, 而断面上的起伏和褶皱是样 品在液氮中断裂所造成的, 这表明 SPES 基体具有 较好的㓞性. 当引入 $\mathrm{AlOOH}$ 后, 表面和断面的形态 发生了较大的变化, 如图 4(b, c) 所示, 复合膜的表面 出现了明显的颗粒状的聚集体, 直径大约在 $2 \mu \mathrm{m}$ 左 右, 且在 SPES/AlOOH 10\%复合膜的表面出现了几 个更大尺寸的聚集体, 这些颗粒状物质应该就是掺 入的 $\mathrm{AlOOH}$ 所形成的聚集物; 对于复合膜的断面而 言, 也出现了类似的颗粒状物质, 但尺寸要比表面 出现的聚集体小, 约在 0.2-0.5 $\mu \mathrm{m}$ 范围内, 这可能 是因为在复合膜的内部, $\mathrm{AlOOH}$ 四周都被磺化聚合 物所包裹, 且 AlOOH 与 SPES 中的极性基团有强烈

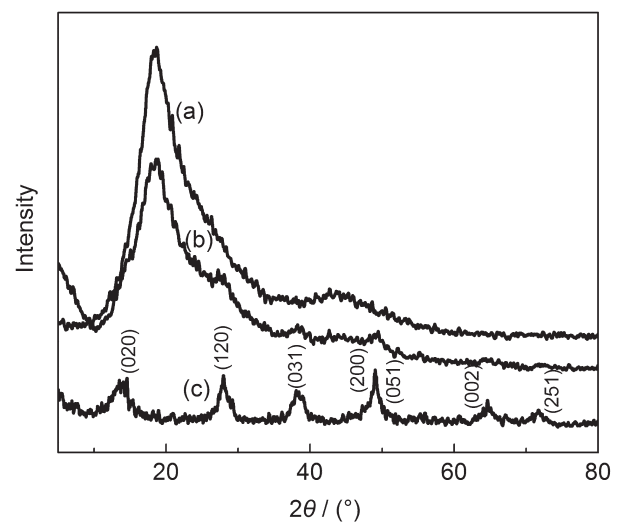

图 3 SPES、AIOOH 与 SPES/AIOOH 复合膜的 XRD 图 Fig.3 XRD patterns of SPES, AIOOH, and SPES/ AlOOH composite membrane (a) SPES, (b) SPES/AlOOH 10\%, (c) $\mathrm{AlOOH}$ 

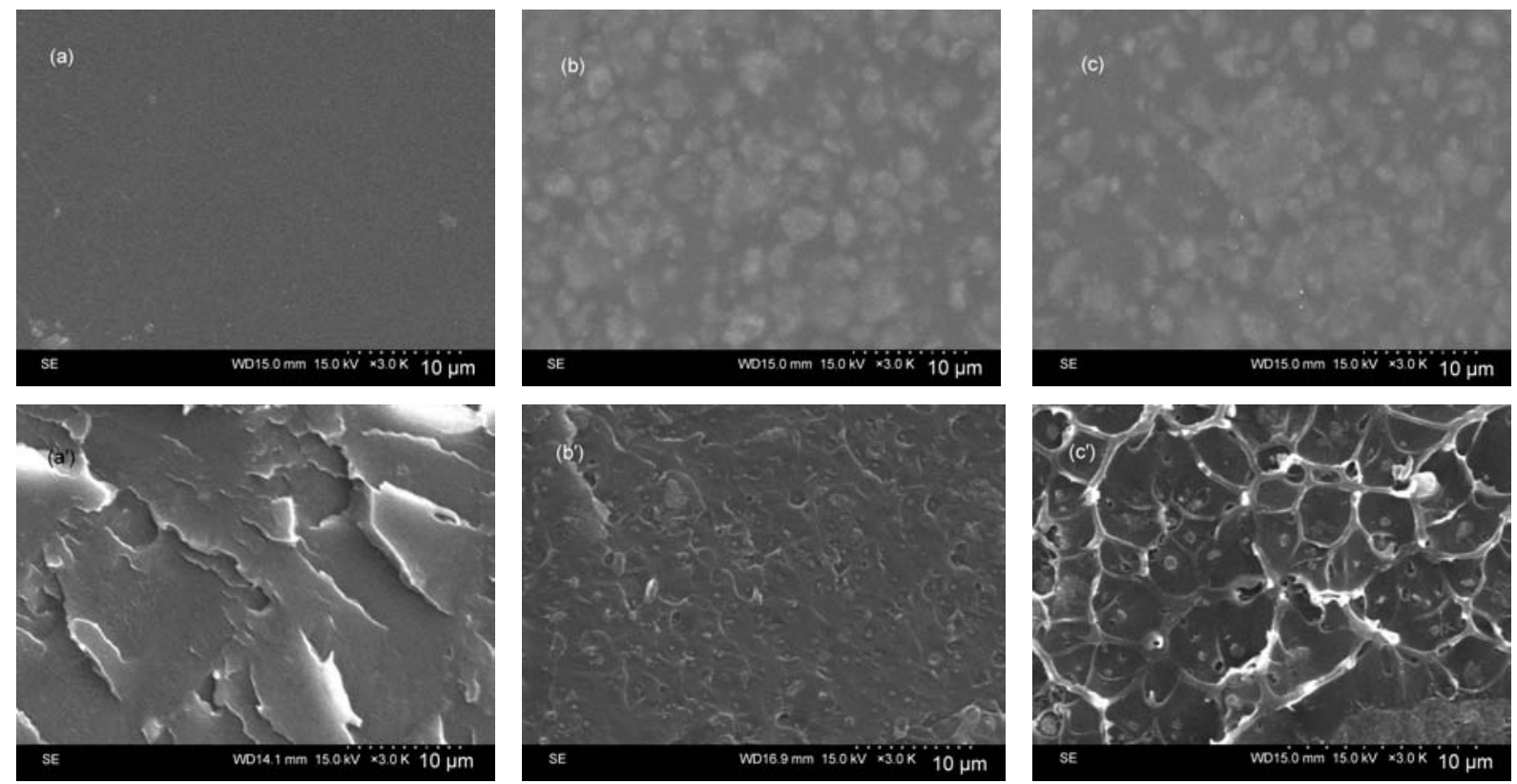

图 4 复合膜的 SEM图

Fig.4 SEM micrographs of the composite membranes

(a) the surface of SPES; $\left(\mathrm{a}^{\prime}\right)$ the cross-section of SPES; (b) the surface of SPES/AlOOH 5\%; (b') the cross-section of SPES/AlOOH 5\%;

(c) the surface of SPES/AlOOH $10 \%$; (c') the cross-section of SPES/AlOOH $10 \%$

的氢键相互作用力, 致使 $\mathrm{AlOOH}$ 之间难以形成大的 聚集体.

为了进一步确认 $\mathrm{AlOOH}$ 在复合膜中的分布情 况，我们对 SPES/AlOOH 10\%复合膜的断面进行了 $\mathrm{EDX}$ 分析, 并重点分析了 $\mathrm{Al}$ 元素的分布, 由图 5(a) 中的结果, $\mathrm{Al}$ 元素占 $4.82 \%(w)$, 经计算得 $\mathrm{AlOOH}$ 与 SPES 的质量比约为 11.8:100, 比实验中所掺杂的量 要高, 这可能是由于仪器测量误差所致. 图 5(b)中白 点分布的位置和密度分别对应于复合膜断面内 $\mathrm{Al}$ 元素的分布的位置和浓度, 由于 $\mathrm{AlOOH}$ 在 $400{ }^{\circ} \mathrm{C}$ 以前只发生吸附水的脱除(见2.2 节), 故 $\mathrm{Al}$ 元素的分 布可以代表 $\mathrm{AlOOH}$ 的分布, 可以看出 $\mathrm{Al}$ 元素的分

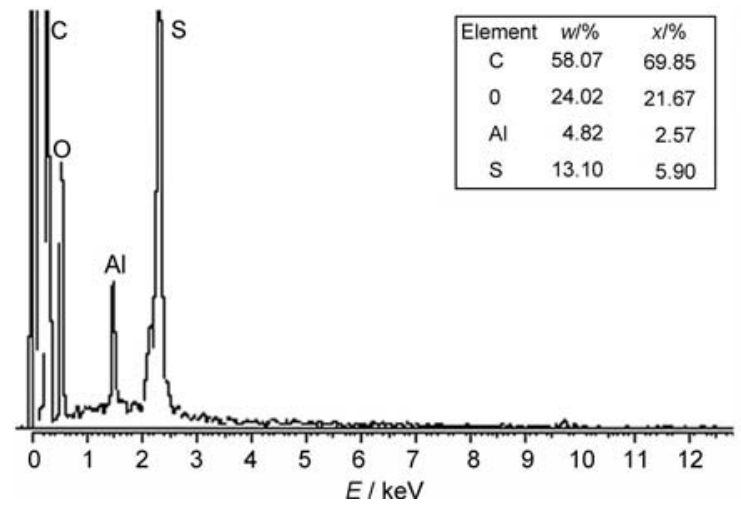

布是较为均匀的, 这也表明 $\mathrm{AlOOH}$ 在复合膜中分散 的很均匀.

\section{5 吸水率和尺寸稳定性}

应用干湿法测定了复合膜在 20 和 $100{ }^{\circ} \mathrm{C}$ 下的 含水率, 如表 1 所示, 可以看出: (1) 在相同温度下, 复合膜的含水率较纯 SPES 膜有了很大程度的提 高, 这得益于 $\mathrm{AlOOH}$ 独特的吸湿性能, 由于 $\mathrm{AlOOH}$ 含有大量的羟基, 这些羟基能够与水分子形成氢 键, 从而使膜吸收大量的水分, 膜的吸水能力随着 $\mathrm{AlOOH}$ 含量的增加而增加, 室温下 SPES/AlOOH $15 \%$ 复合膜的含水率达到 $77.6 \%$, 较纯 SPES 膜增加 了将近 2 倍; (2) 温度升高, 所有膜的含水率均增加,

图 5 SPES/AIOOH 10\%复合膜断面的 X 射线能量色散(EDX)谱

Fig.5 Energy dispersive X-ray (EDX) spectrum of the cross-section of the SPES-AIOOH 10\% composite membrane (a) element distribution; (b) mapping image of Al distribution 
表 1 复合膜的各种物理性质

Table1 Physical properties of composite membranes

\begin{tabular}{|c|c|c|c|c|c|c|c|}
\hline \multirow{2}{*}{ Sample } & \multicolumn{2}{|c|}{ Water uptake/\% } & \multicolumn{3}{|c|}{ Dimensional change/\% } & \multirow{2}{*}{ Oxidative stability/h } & \multirow{2}{*}{$\frac{10^{7} \text { Methanol permeability }}{\left(\mathrm{cm}^{2} \cdot \mathrm{s}^{-1}\right)}$} \\
\hline & $20^{\circ} \mathrm{C}$ & $100^{\circ} \mathrm{C}$ & length & width & thickness & & \\
\hline SPES & 27.1 & 34.5 & 11.7 & 10.4 & 20.2 & 3 & 5.76 \\
\hline SPES/AlOOH 5\% & 36.7 & 49.0 & 12.9 & 10.6 & 22.5 & 4 & 4.12 \\
\hline SPES/AlOOH 10\% & 54.6 & 68.5 & 15.6 & 12.3 & 25.7 & 5.5 & 2.63 \\
\hline SPES/AlOOH 15\% & 77.6 & 90.4 & 20.8 & 14.5 & 29.9 & 8 & 2.89 \\
\hline Nafion 112 & 18.0 & 30.2 & 15.0 & 4.7 & 28.0 & - & 10.5 \\
\hline
\end{tabular}

这是温度升高使水分子运动加剧所致.

复合膜的含水率提高不但有利于磺酸基团上 质子的分离, 而且以水作为质子载体的质子传输过 程也会变得更容易, ${ }^{17}$ 但含水率过高可能会导致膜 溶胀过度, 从而影响其机械性能, 因此平衡膜的含 水率和溶胀性能非常重要. 为此, 我们测定了在 $100{ }^{\circ} \mathrm{C}$ 去离子水中浸泡了 $24 \mathrm{~h}$ 的复合膜的尺寸变 化, 由表 1 所列数据可以看出, 复合膜的溶胀率均高 于纯 SPES 膜, 但对于 SPES/AlOOH 10\%的复合膜 而言, 虽然其含水率远高于 Nafion 112 膜, 但其溶胀 率与 Nafion 112 膜相当, 这可能是由于 $\mathrm{AlOOH}$ 与磺 酸基团间的氢键作用阻止了复合膜的过度溶胀.

\section{6 氧化稳定性}

应用于燃料电池的质子交换膜除了应具有较 好的热稳定性外, 还应具有一定的耐氧化性, 否则 膜在电池使用的过程中会由于发生氧化降解而使 得电池性能大大下降. 采用 Fenton 试剂 (3\% (w) $\left.\mathrm{H}_{2} \mathrm{O}_{2}+4 \times 10^{-6}(\varphi) \mathrm{FeSO}_{4}\right)$ 在 $80^{\circ} \mathrm{C}$ 条件下进行测试, 在 $\mathrm{Fe}^{2+}$ 的催化作用下, 双氧水会分解出 $\mathrm{HO}$ - 和 $\mathrm{HOO}$ - 等 自由基, 这可以模拟燃料电池中的强氧化环境, 以 膜开始溶解的时间作为评价的依据, 实验结果如表 1 所示, 可以看出复合膜的耐氧化性能较纯 SPES 膜 有所增加, 但商用的 Nafion 系列膜具有极其良好的 氧化稳定性, 能在强氧化性溶液中高温长时间浸泡 而不发生溶解, 耐氧化性较差是碳氢芳族聚合物用 作质子交换膜所存在的一个主要问题, 因此有待对 SPES/AlOOH 复合膜的氧化机理进一步研究, 探讨 提高其氧化稳定性的有效方法.

\section{7 质子传导率与甲醇渗透性}

图 6 为不同 $\mathrm{AlOOH}$ 掺杂量的复合膜在不同温 度下的质子传导率. 由图可以看出, 在 $80^{\circ} \mathrm{C}$ 以前, 纯 SPES 膜的质子传导率随着温度的增加而增加, 温度高于 $80^{\circ} \mathrm{C}$ 后, 由于膜内水分的蒸发速度大于 其生成速度而导致膜失水, 质子传导率随之下降; 而对于 SPES/AlOOH复合膜而言, 其质子传导率在
室温下均低于纯 SPES 膜, 且随着 $\mathrm{AlOOH}$ 掺杂量的 增加而降低, 因为 $\mathrm{AlOOH}$ 本身并不能传导质子, 另 外, 质子传导率的下降可能还与组分之间氢键的形 成有关, 因为 $\mathrm{AlOOH}$ 含有较多的羟基, 易与 SPES 中 的磺酸基团等形成分子间氢键, 这样就导致膜内自 由 $-\mathrm{SO}_{3} \mathrm{H}$ 基团数减少, 增加了质子传输的阻力, 使 膜的质子传导率有所降低. 但随着温度的升高, 复 合膜的质子传导率上升, 甚至超过了 SPES 膜的质 子传导率, 且复合膜的质子传导率直到温度超过 $100{ }^{\circ} \mathrm{C}$ 后才开始下降, 对于 $\mathrm{AlOOH}$ 掺杂量为 $5 \%$ 和 $10 \%$ 的复合膜, 在 $100{ }^{\circ} \mathrm{C}$ 时的质子传导率均高于 SPES 膜, 这可能是因为 $\mathrm{AlOOH}$ 具有很强的保水和 滞水能力, 且 $\mathrm{AlOOH}$ 中含有大量的结晶水也可保证 膜内含有足够的水分, 从而可改善膜的质子传导性 能, 尤其是在高温情况下. 由图 6 还可看出, 在同样 的测试条件下, Nafion 112 膜在温度超过 $100{ }^{\circ} \mathrm{C}$ 后, 其质子传导率几乎下降了一半, 这将远不能满足燃 料电池在高温下工作的需求. 而 $\mathrm{SPES} / \mathrm{AlOOH}$ 复合 膜的电导率在温度达到 $100^{\circ} \mathrm{C}$ 后才有所下降, 即使 是 $\mathrm{AlOOH}$ 含量为 $10 \%$ 的复合膜, 其在 $120^{\circ} \mathrm{C}$ 下的质 子传导率仍可保持在 $0.014 \mathrm{~S} \cdot \mathrm{cm}^{-1}$ 左右, 这说明 $\mathrm{SPES} / \mathrm{AlOOH}$ 复合膜在高温下的质子传导率可以满

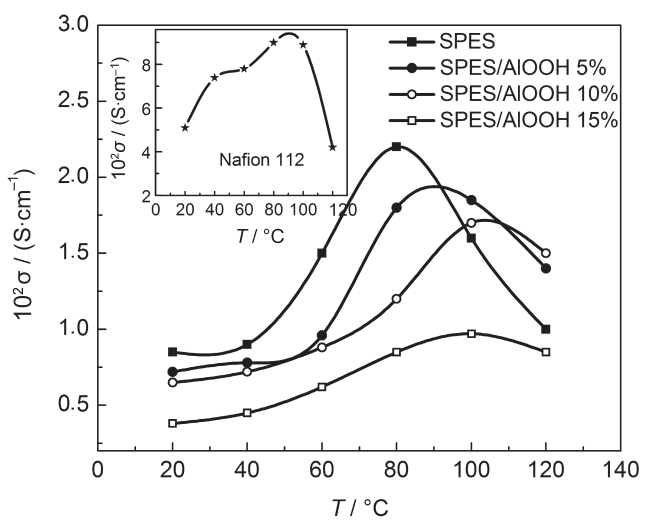

图 6 不同温度下 Nafion 112 膜和复合膜的质子传导率 $(\sigma)$

Fig.6 Proton conductivities $(\sigma)$ of Nafion 112 and composite membranes at different temperatures 
足高温操作的使用要求.

对 DMFC 而言, 质子交换膜的阻醇性能的提高 意味着可采用较高浓度的甲醇作燃料, 从而使 DMFC 的能量密度得到进一步提高. 质子交换膜的 阻醇性能采用甲醇渗透系数 $\left(P_{\mathrm{m}} /\left(\mathrm{cm}^{2} \cdot \mathrm{s}^{-1}\right)\right)$ 来表征. 实验测得商用 Nafion 112 膜的甲醇渗透系数为 $1.05 \times 10^{-6} \mathrm{~cm}^{2} \cdot \mathrm{s}^{-1}$, 与文献报道的基本一致. ${ }^{21}$ 纯 SPES 膜和 SPES/AlOOH复合膜在室温下的甲醇渗 透系数如表 1 所示. 可以看出, SPES 作基体的质子 交换膜的甲醇渗透系数远远低于 Nafion 112 膜, 而 且适量的 $\mathrm{AlOOH}$ 能显著提高膜的阻醇性能, 对 $\mathrm{SPES} / \mathrm{AlOOH} 5 \%$ 的复合膜, 其在室温下 $P_{\mathrm{m}}$ 低至 $4.12 \times 10^{-7} \mathrm{~cm}^{2} \cdot \mathrm{s}^{-1}$, 仅约为 Nafion 112 膜的四分之一. 复合膜的优异阻醇性能可能的原因如下: $\mathrm{AlOOH}$ 对 水有较强的亲和力, 因而在甲醇溶液中, 它首先吸 收水分, 然后再吸收甲醇, 因此可大大降低甲醇的 吸入量. 但同时我们发现, 当 $\mathrm{AlOOH}$ 含量较高时, 阻 醇性有所下降, 这可能是因为 $\mathrm{AlOOH}$ 量过多时, 颗 粒在膜中的分散将会不太均匀, 小的纳米粒子会聚 集成大块颗粒, 而形成微孔, 影响膜的致密性, 改变 膜的网络结构, 使甲醇渗透加快.

\section{4 结 论}

以 SPES 为基体制备了一种新型的 SPES/ $\mathrm{AlOOH}$ 复合膜, 得到了预期的化学和物理结构. 随 着 $\mathrm{AlOOH}$ 的引入, 复合膜的质子传导率虽有所下 降, 但在高温下 $\left(100^{\circ} \mathrm{C}\right)$ 质子传导率要高于纯 SPES 膜. SPES 与 $\mathrm{AlOOH}$ 的复合可有效降低膜的甲醇渗 透性能, $\mathrm{AlOOH}$ 掺杂量为 $10 \%$ 时, 可使复合膜的甲 醇渗透系数较 Nafion 112 膜降低将近一个数量级, 同时复合膜的热稳定性也得到了提高, 有希望作为 质子交换膜而用于高温 DMFC 中.

\section{References}

(1) Fu, X. Z.; Li, J.; Lu, C. H.; Liao, D. W. Progress in Chemistry 2004, 16 (1), 77 . [符显珠, 李 俊, 卢成慧, 廖代伟. 化学进 展, 2004, $16(1), 77$.

(2) Gong, C. L.; Zhou, Y.; Yan, L. C.; Wen, S.; Zheng, G. W. Acta Phys. -Chim. Sin. 2010, 26 (11), 2967. [龚春丽, 周 毅, 间礼 成, 文 胜, 郑根稳. 物理化学学报, 2010, 26 (11), 2967.]

(3) Deng, H. N.; Li, L.; Xu, L.; Wang, Y. X. Acta Phys. -Chim. Sin.
2007, 23 (8): 1235. [邓会宁, 李 否, 许 莉, 王宇新. 物理化 学学报, 2007, 23 (8), 1235.]

(4) Rhee, C. H.; Kim, H. K.; Chang, H.; Lee, J. S. Chem. Mater. 2005, 17, 1691.

(5) Yang, S. F.; Gong, C. L.; Guan, R.; Zou, H.; Dai, H. Polym. Adv. Technol. 2006, 17, 360.

(6) Yi, B. L. Fuel Cells—Principle -Technology•Application; Chemical Industry Press: Beijing, 2003; p 351. [衣宝廉. 燃料 电池一原理.技术.应用. 北京: 化学工业出版社, 2003: 351.]

(7) Nolte, R.; Ledjeff, K.; Bauer, M.; Mülhaupt, R. J. Membr. Sci. 1993, 83, 211.

(8) Guan, R.; Dai, H.; Li, C.; Liu, J.; Xu, J. J. Membr. Sci. 2006, $277,148$.

(9) Kopitzke, R. W.; Linkous, C. A.; Anderson, H. R.; Nelson, G. L. J. Electrochem. Soc. 2000, 147, 1677.

(10) Dai, H.; Guan, R.; Li, C.; Liu, J. Solid State Ionics 2007, 178, 339.

(11) Tsai, J. C.; Kuo, J. F.; Chen, C. Y. J. Power Sources 2007, 174, 103.

(12) Su, Y. H.; Liu, Y. L.; Sun, Y. M.; Lai, J. Y.; Wang, D. M.; Gao, Y.; Liu, B.; Guiver, M. D. J. Membr. Sci. 2007, 296, 21.

(13) Nunes, S. P.; Ruffmann, B.; Rikowski, E.; Vetter, S.; Richau, K. J. Membr. Sci. 2002, 203, 215.

(14) Jin, H.; Xie, X. F.; Shang, Y. M.; Feng, S. G.; Lü, Y. F. Chemical Industry and Engineering Progress 2007, 26 (4), 507. [靳 豪, 谢晓峰, 尚玉明, 冯少广, 吕亚非. 化工进展, 2007, 26 (4), 507.]

(15) Shen, J.; Xi, J. Y.; Zhu, W. T.; Chen, L. Q.; Qiu, X. P. J. Power Sources 2006, 159, 894.

(16) Yan, J. S.; Long, J.; Tian, H. P. Petroleum Processing and Petrochemicals 2004, 35 (9), 38. [严加松, 龙 军, 田辉平. 石油炼制与化工, 2004, 35 (9), 38.]

(17) Ye, T. X.; Liu, J. Y.; Zhang, B.; Ma, X. N. Chinese Journal of Applied Chemistry 2009, 26 (9), 1114. [叶天旭, 刘京燕, 张 斌, 马雪妮. 应用化学, 2009, 26 (9), 1114.]

(18) Lü, J. G.; Zhang, J.; Ding, W. P.; Shen, B.; Guo, X. F. Chinese Journal of Inorganic Chemistry 2007, 23 (5), 897. [吕建刚, 张 娟, 丁维平, 沈 樹, 郭学峰. 无机化学学报, 2007, 23 (5), 897.]

(19) Liu, C. H.; Liao, H. D.; Long, X. Y. Journal of Southwest China Normal University 2003, 28 (2), 263. [刘昌华, 廖海达, 龙翔 云. 西南师范大学学报(自然科学版), 2003, 28 (2), 263.]

(20) Zheng, G. W.; Gong, C. L.; Wen, S.; Zhou, H. B.; Xie, X. L. Acta Phys. -Chim. Sin., 2009, 25 (3), 575. [郑根稳, 龚春丽, 文 胜, 周环波, 解孝林. 物理化学学报, 2009, 25 (3), 575.]

(21) Shu, Y. C.; Chuang, F. S.; Tsen, W. C.; Gong, C. L.; Wen, S. J. Appl. Polym. Sci. 2008, 108, 1783. 